\title{
Actividade física, equilíbrio e medo de cair. Um estudo em idosos institucionalizados
}

\author{
Joana Carvalho \\ Joana Pinto \\ Jorge Mota
}

\author{
Centro de Investigação em Actividade Física, \\ Saúde e Lazer \\ Faculdade de Desporto \\ Universidade do Porto \\ Portugal
}

\section{RESUMO}

O objectivo deste trabalho foi determinar qual a relação entre o medo de cair, o equilíbrio e a prática de actividade física em idosos institucionalizados.

A amostra foi constituída por 56 idosos institucionalizados (65 e 95 anos) divididos em função do padrão de prática de actividade física e do sexo. O equilíbrio, o medo de cair e a ocorrência de quedas nos últimos 12 meses foram estudados como factores de risco para as quedas. Os resultados mostraram que: i) não se verificaram diferenças estatisticamente significativas no equilíbrio em função do sexo; ii) os idosos do sexo masculino apresentaram menor medo de cair que os do sexo feminino; iii) os praticantes de actividade física apresentaram maior equilíbrio e menor medo de cair do que os não praticantes; iv) verificou-se uma associação positiva entre o medo de cair e o equilíbrio, entre o medo de cair e a prática de actividade física e entre o equilíbrio e a prática de actividade física. Conclui-se que, em idosos institucionalizados, o sexo não influencia o equilíbrio mas influencia negativamente o medo de cair e que a ocorrência de quedas não parece ter grande impacto no medo de cair. Os resultados deste trabalho sugerem ainda que a prática de actividade física está associada a um maior equilíbrio e a um menor medo de cair.

Palavras-chave: envelhecimento, quedas, equilíbrio, medo de cair, actividade física.

\section{ABSTRACT \\ Physical activity, balance and fear of falling. A study with institutionalized older people}

The aim of the present study was to investigate the relationship between fear of falling, balance and physical activity in institutionalized older adults.

Fifty six subjects aged 65 or older living in nursing homes participated in this study. Balance, fear of falling and the occurrence of falls during the last 12 months were assessed as risk factors for falls. The results showed: (i) no significant differences in balance according to gender; (ii) men reported less fear of falling than women; (iii) active elderly subjects showed significantly higher values of balance and less fear of falling compared to non-participants peers; (iv) a positive association was observed between fear of falling and balance, between fear of falling and physical activity and between balance and physical activity. We concluded that gender did not influence balance but negatively affects fear of falling. Participation in physical activity programs seems to be associated and to have positive effects on balance and fear of falling.

Key-words: aging, falls, balance, fear of falling, physical activity 


\section{INTRODUÇÃO}

A população idosa é, actualmente, uma realidade demográfica cada vez mais significativa na população mundial. Por exemplo, em Portugal, entre 1960 e 2001, o fenómeno do envelhecimento demográfico traduziu-se por um incremento de $140 \%$ da população idosa com decréscimo de cerca de $36 \%$ na população jovem(12).

O número de idosos institucionalizados tem vindo a aumentar, quer em lares, centros de dia ou casas de repouso. Em Portugal, cerca de $33 \%$ dos utentes ligados a estabelecimentos de segurança social, são idosos, nomeadamente $12 \%$ em lares, $11 \%$ em apoio domiciliário e $10 \%$ em centros de dia(12).

Viver na comunidade ou em instituições são duas condições de vida distintas geralmente associadas a diferenças nos índices de actividade física e níveis de incapacidade ${ }^{(10)}$. Apesar de, muitas vezes, os idosos serem institucionalizados ainda com um nível de autonomia bastante elevado, a habitual desobrigação da realização de várias das tarefas do dia a dia neste contexto, contribui para o aumento da inactividade, para a redução da aptidão física e consequentemente, para o aumento do risco de quedas, da morbilidade e mortalidade(10).

As quedas são um dos mais sérios problemas associados com a idade e um dos maiores problemas de Saúde Pública(3,13). Vários trabalhos mostram que 40 a $60 \%$ dos indivíduos acima dos 65 anos já experimentaram pelo menos uma queda, sendo esta mais frequente nos utentes de lares e nas mulheres (23). São vários os factores relacionados com a maior susceptibilidade de ocorrência de quedas e consequentes fracturas, facilitadas pela desmineralização óssea comum neste escalão etário( ${ }^{(3,6)}$. Entre outros, a diminuição do equilíbrio e o medo de cair são aspectos determinantes em todo este quadro(3). Estudos como os de Tinetti et al.(29) e MacAuley et al.(16) referem o medo de cair como um factor psicológico presente em $50 \%$ das pessoas idosas reportando uma experiência prévia de queda.

As alterações do equilíbrio e o medo de cair afectam a auto-confiança, repercutindo-se negativamente na quantidade de actividade física diária, nos níveis de aptidão física e no envolvimento das actividades da vida diária (AVD), factos estes que por sua vez contribuem para o isolamento social e aumento da dependência de outrem $(14,27,3)$. Assim, parece existir um ciclo vicioso negativo entre a inactividade, o fraco equilíbrio, o medo de cair e a maior probabilidade de ocorrência de quedas(3).

A prática de actividade física regular tem sido referida como uma importante e eficaz estratégia de prevenção das quedas(15) ao promover o aumento dos níveis de aptidão física e de auto-confiança na realização de tarefas do dia a dia, particularmente nos idosos com maior grau de incapacidade ${ }^{(4)}$.

Tomando em consideração o atrás exposto, o objectivo deste estudo consistiu em determinar qual a relação entre o medo de cair, o equilíbrio e a prática de actividade física em idosos institucionalizados. Para além disso, este trabalho procurou analisar a influência das variáveis sexo e prática de actividade física regular sobre o equilíbrio e o medo de cair. Por fim, pretendeu-se observar de que forma é que a história de quedas nos últimos doze meses influenciam o medo de cair.

\section{MATERIAL E MÉTODOS Amostra}

Participaram voluntariamente 58 voluntários residentes em 2 lares da região de Guimarães. A presença de patologias crónicas e o uso de medicamentos foram obtidos através de informação reportada pelos sujeitos da amostra e pelo médico assistente do corpo clínico das instituições a que pertenciam, mediante autorização prévia dos idosos.

Pelas próprias características dos sujeitos, a amostra inicial foi alterada de forma a serem controladas todas as variáveis que poderiam influenciar nos resultados finais. Assim, foram excluídos 2 indivíduos, um por apresentar problemas de visão e outro pelo facto de se encontrar sob efeito farmacológico de anti-depressivos.

Assim, a amostra final passou a ser constituída por 56 indivíduos com idades compreendidas entre os 65 e os 95 anos (idade média $=77.5 \pm 7.8$ anos), dos quais 32 do sexo feminino e 24 do sexo masculino. A avaliação da prática regular de actividade física estruturada (no período de 12 meses prévios ao inicio do presente estudo) para posterior classificação e alocação dos idosos em grupos, foi efectuada através de um questionário que incluiu questões referentes ao tipo de atividade física praticada, a freqüência 
semanal de tal prática e a duração das sessões de treinamento. Consideraram-se praticantes os indivíduos que participavam regularmente em, pelo menos, sessões bissemanais de actividade física estruturada, com uma duração de pelo menos 50 minutos e dirigidas para várias componentes de aptidão física (treino de resistência aeróbia, força muscular, equilíbrio, flexibilidade e coordenação).

Os idosos foram assim subdivididos em dois grupos em função dos hábitos de prática actividade física regular: $\mathrm{P}$ - praticantes de actividade física envolvendo 28 sujeitos com idades compreendidas entre os 65 e os 92 anos (idade média $=77.1 \pm 7.2$ anos), dos quais 16 mulheres e 12 homens e NP - não praticantes de actividade física compreendendo 28 indivíduos com idades compreendidas entre os 65 e os 95 anos (idade média $=79.4 \pm 8.1$ anos), dos quais 16 mulheres e 12 homens.

Através do questionário determinou-se, também, a ocorrência de quedas no período de doze meses anteriores ao estudo.

Foram considerados os aspectos éticos referidos na Declaração de Helsínquia (1986) da Associação Médica Mundial, incluindo a adequada informação dos participantes em relação ao estudo, a manutenção da sua confidencialidade e anonimato através da codificação dos participantes, assim como, a obtenção do seu consentimento informado escrito antes de se efectuar a recolha de dados.

\section{Avaliação do medo de cair}

Para avaliar o medo de cair utilizou-se a versão portuguesa da Falls Efficacy Scale (FES). Este instrumento foi desenvolvido para medir o medo de cair por Tinetti et al.(29), tendo sido validado para a população portuguesa por Melo(17). É baseado na definição operacional de medo como "percepção de diminuta auto-confiança para evitar quedas durante tarefas essenciais, potencialmente não lesivas"(29). Esta escala mede o medo de cair, perguntando-se ao individuo qual o grau de confiança que tem na realização de determinadas tarefas sem cair ou perder o equilíbrio. É constituída por um questionário com 10 tarefas (vestir e despir; preparar uma refeição ligeira; tomar um banho ou duche; sentar/levantar da cadeira; deitar/levantar da cama; atender a porta ou o telefone; chegar aos armários; trabalho doméstico ligeiro; fazer pequenas compras) que são avaliadas numa escala de 10 pontos, onde o zero (0) representa nenhuma confiança e o 10 muito confiante.

Assim, após somatório dos valores, aqueles mais baixos significam pouca confiança ou maior medo de cair, e aqueles mais altos significam muita confiança ou menor medo de cair.

\section{Avaliação do equilíbrio}

Para a avaliação do equilíbrio utilizou-se a versão portuguesa da Performance-Oriented Mobility Assessment (POMA I). Este instrumento foi desenvolvido por Tinetti(28) e validado para a população portuguesa por Petiz(21). Estima a predisposição para quedas em idosos institucionalizados através da avaliação quantitativa de um conjunto de tarefas relacionadas com a mobilidade e o equilíbrio.

Está dividido em duas partes que totalizam 28 pontos onde quanto mais alto o valor melhor o equilíbrio. A primeira parte diz respeito à avaliação do equilíbrio estático, com 9 itens, dos quais dois são pontuáveis de 0 a 1 e sete de 0 a 2 , permitindo um máximo de 16 pontos. A segunda parte avalia o equilíbrio dinâmico e envolve 10 itens dos quais oito são pontuáveis de 0 a 1 e dois de 0 a 2 num total de 12 pontos.

De acordo com Petiz(21), a versão portuguesa da POMA I apresenta elevada homogeneidade de conteúdo $(\alpha=0,97)$ e fiabilidade após teste-reteste $(r$ de Pearson $=0,96)$. A validade de critério deste teste de equilíbrio foi, também, estudada, tendo sido utilizados, para o efeito, o functional reach test e o timed up and go test, para o equilíbrio estático e dinâmico, respectivamente(21). A autora descreveu elevadas correlações entre os testes anteriormente referidos, comprovando a validade de critério, quer da sub-escala de equilíbrio estático $(r=0,78)$, quer do equilíbrio dinâmico $(r=0,89)$.

\section{Procedimentos estatísticos}

A descrição das variáveis em estudo foi efectuada a partir das medidas descritivas média e desviopadrão.

Procedeu-se a uma análise exploratória dos dados (Shapiro-Wilk) com o objectivo de averiguar a normalidade da distribuição correspondente a cada uma das variáveis em estudo, assim como a presença de 
"outliers". A análise das diferenças entre os grupos foi efectuada a partir do t-teste de medidas independentes e do teste Mann-Whitney. De forma a averiguar o grau de correlação existente entre as variáveis medo de cair, equilíbrio e prática de actividade física utilizamos o coeficiente de correlação de Spearman. Foi seleccionado um nível de significância de $5 \%$.

\section{RESULTADOS}

Influência do sexo no equilíbrio e medo de cair O quadro 1 representa os valores relativos ao teste de equilíbrio (POMA) e ao medo de cair (FES) de acordo com a variável sexo.

$\begin{array}{lcc} & \begin{array}{c}\text { Quadro 1. Valores médios do equilíbrio e medo de cair } \\ \text { em função do sexo (média } \pm \text { desvio padrão). }\end{array} \\ \begin{array}{lcc} \\ \text { Sexo } & \text { Equilíbrio } & \text { Medo de cair } \\ \hline \text { Homens } & 23.5 \pm 4.15 & 68,79 \pm 15,99 * \\ \text { Mulheres } & 20.94 \pm 6.54 & 51,72 \pm 23,18 \\ \hline \multicolumn{4}{c}{\text { *Homens vs. Mulheres }(p<0.05)}\end{array}\end{array}$

Podemos constatar que não foram encontradas diferenças com significado estatístico entre homens e mulheres no equilíbrio (Mann-Whitney $\mathrm{U}=303.50$, $\mathrm{p}=0.18$ ), apresentando, no entanto, os homens pontuações médias na FES significativamente superiores comparativamente com as mulheres (Mann-Whitney $\mathrm{U}=225, \mathrm{p}=0.01$ ), sugerindo que estas apresentam um maior medo de cair.

\section{Influência da actividade física no equilíbrio e medo de cair}

No quadro 2 podemos visualizar a comparação entre idosos praticantes e não praticantes de actividade física relativamente ao equilíbrio e medo de cair.

Quadro 2. Valores médios do equilíbrio e medo de cair em função da prática de actividade física [média \pm desvio padrão].

\begin{tabular}{lcc}
\hline & Praticantes & Não praticantes \\
\hline Equilíbrio & $25.68 \pm 2.78$ & $18.39 \pm 5.65$ \\
Medo de cair & $69.14 \pm 16.45$ & $48.93 \pm 22.37$ \\
\hline
\end{tabular}

A análise do quadro anterior mostra que os valores médios do POMA e FES dos praticantes de actividade física são significativamente superiores aos dos não praticantes $(\mathrm{t}(39.34)=-6.13, \mathrm{p}<0.001$ e $\mathrm{t}(54)=-$ $3.85, \mathrm{p}<0.001$, respectivamente), sugerindo que os idosos que praticam actividade física têm maior equilíbrio e menor medo de cair comparativamente aos não praticantes.

\section{Relação entre o medo de cair, equilíbrio e prática de actividade física}

O quadro 3 representa a matriz de correlação das diferentes variáveis analisadas.

Quadro 3. Matriz de correlação entre o equilíbrio, o medo de cair e a prática de actividade física.

\begin{tabular}{lccc}
\hline & Medo de cair & Equilíbrio & Actividade física \\
\hline Medo de cair & 1 & $0.76^{* *}$ & $0.47^{* *}$ \\
Equilíbrio & $0.76^{* *}$ & 1 & $0.67^{* *}$ \\
Actividade Física & $0.47^{* *}$ & $0.67^{* *}$ & 1 \\
\hline \multicolumn{4}{c}{${ }^{* *} p<0.01$}
\end{tabular}

Verifica-se uma associação positiva e estatisticamente significativa $(r=0.76 ; \mathrm{p}<0.01)$ entre as variáveis medo de cair (pontuação da FES) e equilíbrio (pontuação do POMA). É possível verificar que um aumento ao nível da pontuação da FES, que indicia um menor medo de cair, é, tendencialmente, acompanhado por um maior equilíbrio. Constata-se a existência de uma associação positiva e estatisticamente significativa $(r=0.47 ; \mathrm{p}<0.01)$, entre as variáveis medo de cair e prática de actividade física. Existe, igualmente, uma associação positiva e estatisticamente significativa $(r=0.67 ; \mathrm{p}<0.01)$ entre as variáveis equilíbrio e a prática de actividade física. O quadro 4 apresenta os resultados obtidos na FES, relativos ao medo de cair, em função da história de quedas anteriores (últimos 12 meses).

Quadro 4. Medo de cair em função da história de quedas (média \pm desvio padrão].

\begin{tabular}{lll}
\hline Ocorrência de quedas & $\mathbf{n}$ & Medo de cair \\
\hline Sim & 21 & $49.33 \pm 22.87$ \\
Não & 35 & $64.86 \pm 19.49$ \\
\hline
\end{tabular}

Podemos constatar que não foram encontradas diferenças com significado estatístico no medo de cair em função da história de quedas recentes. 


\section{DISCUSSÃO}

Um dos objectivos deste trabalho foi identificar o efeito da variável género sobre o equilíbrio e medo de cair em sujeitos idosos institucionalizados como factor importante para o risco de quedas. Os resultados deste estudo mostraram não existirem diferenças entre homens e mulheres na capacidade de equilíbrio. Embora a maioria dos estudos descreva a existência de diferenças no equilíbrio entre homens e mulheres, a ausência de diferenças observadas no presente estudo têm também sido descritas por outros autores. Por exemplo, Hageman et al.(9) não relataram nenhuma diferença entre homens e mulheres idosos (60-75 anos) nas medidas de controlo postural observadas, quer mediante a utilização de uma plataforma de forças, quer no teste de "functional reach". Mais recentemente, Musselman e Brouwer(19) também não encontraram diferenças entre géneros nos limites de estabilidade. Contudo, a comparação destes resultados com estudos anteriores é, de certo modo, limitada dadas as diferenças nas metodologias, amostra e protocolos utilizados. Os estudos que reportam diferenças entre homens e mulheres na capacidade de equilíbrio justificam os seus resultados, quer em factores músculo-esqueléticos nomeadamente ao nível dos membros inferiores(5), quer nos diferentes padrões de actividade físi$\mathrm{Ca}^{(32)}$. Assim, e considerando que os idosos da nossa amostra são institucionalizados, onde as diferenças nos índices de actividade física em função do género não são tão evidentes, este facto poderá justificar os nossos resultados. De facto, nenhum dos estudos que refere o maior equilíbrio dos homens foi efectuado em idosos institucionalizados ao contrário da presente investigação.

No que respeita ao medo de cair, os resultados do nosso estudo estão de acordo com diferentes trabalhos que referem que as mulheres idosas apresentam mais medo de cair do que os homens idosos $(14,25)$. Autores como Tinetti et al.(30) e McAuley et al.(16) sugerem que o menor medo de cair nos indivíduos do sexo masculino comparativamente aos do sexo feminino, poderá estar relacionado com o facto de os homens não reconhecerem esse medo, evitando um potencial estigma. De facto, a quantificação do medo de cair, através da escala FES, depende da resposta dos indivíduos em estudo a uma série de questões e, consequentemente, os resultados poderão ser manipulados por estes, no caso de não responderem com sinceridade às perguntas que lhes são colocadas. Para além destes factores, alguns estudos justificam o facto de as mulheres apresentarem um maior medo de cair dado o seu maior declínio na funcionalidade do sistema muscular esquelético comparativamente aos homens $(18,14)$.

Outro dos objectivos do presente estudo está relacionado com a influência da actividade física formal sobre o equilíbrio e o medo de cair dos idosos institucionalizados. Os nossos resultados sugerem que os idosos que praticam actividade física apresentam melhores resultados no POMA, ou seja, melhor mobilidade e equilíbrio, quando comparados com aqueles que não praticam actividade física. Este facto está de acordo com a literatura, onde a maioria dos estudos sugere que a prática de actividade física regular é eficaz no aumento de equilíbrio $(20,1,7)$ e que, pelo contrário, um estilo de vida sedentário associado com o envelhecimento leva à sua consequente diminuição(24). Isto significa que, as estratégias que induzam melhorias a este nível são fundamentais para a prevenção de quedas. O equilíbrio é, então, uma capacidade que deverá ser desenvolvida nestes escalões etários mais velhos, particularmente naqueles mais inactivos e mais incapacitados como é o caso da maioria dos idosos institucionalizados ${ }^{(10)}$. No que diz respeito ao medo de cair, e em concordância com estudos anteriores $(16,26,2,22)$ observamos no presente estudo que os indivíduos praticantes de actividade física apresentavam valores mais altos da FES, isto é, menor medo de cair comparativamente com aqueles que não praticavam actividade física. Recentemente, Schoenfelder e Rubenstein ${ }^{(22)}$ verificaram que um programa de exercícios com duração de três meses induziu melhorias significativas no equilíbrio e na diminuição do medo de cair avaliada, tal como no presente estudo, através da escala da FES. Taggart(26) obteve resultados semelhantes, tendo demonstrado o efeito benéfico observado pelo aumento do equilíbrio e uma diminuição do medo de cair que um programa de actividade física baseado no Tai Chi teve em mulheres idosas institucionalizadas. O medo de cair é comum entre os idosos, particularmente entre os idosos institucionalizados, sendo considerado como um factor de risco independente para 
a redução da mobilidade e da qualidade de vida $(2,8)$. Neste sentido, o medo da queda é uma barreira psicológica importante que necessita de ser vencida de forma a favorecer estilos de vida mais activos.

No presente trabalho foi possível verificar a existência de uma associação positiva e significativa entre a pontuação da FES, que indicia um menor medo de cair, com um maior equilíbrio.

Suzuki et al.(25) verificaram que ter muito medo de cair estava fortemente associado não apenas com uma diminuição do equilíbrio mas igualmente com a redução da mobilidade, da actividade física e com o aumento de quedas.

De igual modo, Brouwer et al.(2) sugerem que indivíduos que apresentam reduzidos níveis de equilíbrio apresentam uma elevada preocupação com a ocorrência de quedas e pouca confiança no seu próprio equilíbrio, limitando, por consequência, as suas actividades. Ou seja, existe um ciclo vicioso onde a inactividade acentua a perda funcional determinante na manutenção do equilíbrio postural e, por seu lado, esta perda de equilíbrio e o medo de cair restringem a actividade quotidiana do idoso(2).

A actividade física, pelo contrário, ao influenciar positivamente capacidades como a força muscular e o equilíbrio e reduzir o medo de cair poderá ajudar a "quebrar" este ciclo, devolvendo a funcionalidade, a autonomia e a qualidade de vida aos idosos(15).

Por fim no nosso estudo não foram encontradas diferenças com significado estatístico no medo de cair entre idosos com e sem história de quedas recentes (12 meses). Tinetti e Williams(31) relatam uma prevalência do medo de cair em cerca de $50-60 \%$ dos idosos com história de queda recente e $20-46 \%$ naqueles que não tem história de queda. Em oposição ao nosso estudo, diversos estudos retrospectivos demonstram que, após ocorrências de uma queda, se desenvolve um maior medo de cair(11, 14). Todavia, escasseiam estudos prospectivos que determinem com clareza qual o risco de ocorrência de quedas em idosos que apresentem medo de cair.

Os resultados deste estudo sugerem que: i) apesar dos homens apresentarem menor medo de cair, não existem diferenças na capacidade de equilíbrio entre homens e mulheres; ii) a prática de actividade física está associada a um maior equilíbrio e menor medo de cair; iii) a história de quedas recentes não influência sobremaneira o medo de cair de idosos institucionalizados.

O presente estudo reforça a importância da prática de actividade física como uma estratégia preventiva para a menor ocorrência de quedas, uma vez que parece influenciar positivamente o equilíbrio e o medo de cair em idosos institucionalizados.

\section{CORRESPONDÊNCIA Joana Carvalho}

Faculdade de Desporto

Universidade do Porto

Rua Plácido Costa, 91

4200 Porto, Portugal

Telefone: 351-22-5074785

Fax: 351-22-5500689

e-mail: jcarvalho@fade.up.pt 


\section{REFERÊNCIAS}

1. Barnett A, Smith B, Lord SR, Williams M, Baumand A (2003). Community-based group exercise improves balance and reduces falls in at-risk older people: a randomised controlled trial. Age Ageing 32: 407-414.

2. Brouwer BJ, Walker C, Rydahl SJ, Culham EG (2003). Reducing fear of falling in seniors through education and activity programs: a randomized trial. J Am Geriatr Soc 51(6): 829-34

3. Carter ND, Kannus P, Khan KM (2001). Exercise in the prevention of falls in older people. Sports Med 31(6): 427-438.

4. Carvalho J, Santos C, Mota J (2005). Actividade física aptidão física de idosos institucionalizados e não-institucionalizados. Arquivos de Fisiatria e Doenças Osteo-articulares 46: 35-43.

5. Daubney ME, Culham EG (1999). Lower-extremity muscle force and balance performance in adults aged 65 years and older. Phys Ther 79(12): 1177-85.

6. Edelberg HK (2001). Falls and function. How to prevent falls and injuries in patients with impaired mobility. Geriatrics 56: 41-45.

7. Federici A, Bellagamba S, Rocchi MB (2005). Does dancebased training improve balance in adult and young old subjects? A pilot randomized controlled trial. Aging Clin Exp Res 17(5): 385-389.

8. Fletcher PC, Hirdes JP (2004). Restriction in activity associated with fear of falling among community-based seniors using home car services. Age Aging 33(3): 273-279.

9. Hageman PA, Leibowitz JM, Blanke D (1995). Age and gender effects on postural control measures. Arch Phys Med Rehabil 76(10): 961-965.

10. Henry C, Webster-Gandy J, Varakamin C (2001). A comparison of physical activity levels in two contrasting elderly populations in Thailand. Am J Human Biol 13: 310.

11. Howland J, Lachman ME, Peterson EW, Cote J, Kasten L, Jette A (1998). Covariates of fear of falling and associated activity curtailment. Gerontologist 38(5): 549-55.

12. Instituto Nacional de Estatística (2002). O envelhecimento em Portugal: situação demográfica e sócio-económica recente das pessoas idosas. Serviço de Estudos sobre a população do Departamento de Estatísticas Censitárias e da população.

13. Kannus P, Parkkari J, Niemi S, Palvanen M (2005). Fallinduced deaths among elderly people. Am J Public Health 95(3):422-424.

14. Lachman ME, Howland J, Tennstedt S, Jette A, Assmann S, Peterson EW (1998). Fear of falling and activity restriction: the survey of activities and fear of falling in the elderly (SAFE). J Gerontol 53(1): P43-50.

15. Lord SR, Castell S, Corcoran J, Dayhew J, Matters B, Shan A, Williams P (2003). The effect of group exercise on physical functioning and falls in frail older people living in retirement villages: a randomized, controlled trial. J Am Geriatr Soc 51(12): 1685-92.

16. McAuley E, Mihalko SL, Rosengren K (1997). Self-efficacy and balance correlates of fear of falling in the elderly. JAPA 5: 329-340.
17. Melo C (2003). Adaptação cultural e validação para a população portuguesa da Falls Efficacy Scale (FES). Trabalho apresentado no âmbito da apresentação de provas públicas para Professora Coordenadora da Escola Superior e Tecnologia da Saúde do Porto (ESTES-Porto), não publicado, ESTESPorto.

18. Myers AM, Powell L, Maki BE, Holliday PJ, Brawley LR, Sherk W (1996). Psychological indicators of balance confidence: relationship to actual perceived abilities. J Gerontol 51: M37-M43.

19. Musselman K, Brouwer B (2005). Gender-related differences in physical performance among seniors. J Aging Phys Act 13(3):239-53.

20. Perrin PP, Gauchard GC, Perrot C, Jeandel C (1999). Effects of physical and sporting activities on balance control in elderly people. Br J Sports Med 33: 121-126.

21. Petiz EM (2002) A actividade física, equilíbrio e quedas. Um estudo em idosos institucionalizados. Tese de Mestrado, não publicada, Faculdade de Ciências do Desporto e de Educação Física da Universidade do Porto.

22. Schoenfelder DP, Rubenstein LM (2004). An exercise program to improve fall-related outcomes in elderly nursing home residents. Appl Nurs Res 17(1): 21-31.

23. Shephard RJ (1990). Exercise for the frail elderly. Sports Training Med Rehabil 1: 263-277.

24. Skelton DA, Beyer N (2003). Exercise and injury prevention in older people. Scand J Med Sci Sports13(1):77-85.

25. Suzuki M, Ohyama N, Yamada K, Kanamori M (2002). The relationship between fear of falling, activities of daily living and quality of life among elderly individuals. Nursing and Health Sciences 4: 155-161.

26. Taggart HM (2002). Effects of Tai Chi exercise on balance, functionalmobility, and fear of falling among older women. Appl Nurs Res 15(4): 235-42.

27. Tennstedt S, Howland J, Lachman M, Peterson E, Kasten L, Jette A (1998). A randomized, controlled trial of a group intervention to reduce fear of falling and associated activity restriction in older adults. J Gerontol 53(6): P38492.

28. Tinetti ME (1986). Performance-Oriented Assessment of Mobility Problems in the elderly patients. JAGS 34: 119126.

29. Tinetti ME, Richman D, Powell L (1990). Falls efficacy as a measure of fear of falling. J Gerontol 45(6): P239-43.

30. Tinetti ME, Mendes de Leon CF, Doucette JT, Baker DI (1994). Fear of falling and fall-related efficacy in relationship to functioning among community-living elders. $J$ Gerontol 49(3): M140-7.

31. Tinetti ME, Williams CS (1998). The effect of falls and falls and fall infuries on functioning in community-dwelling older persons. J Gerontol 53(2): 112-119.

32. Wojcik LA, Thelen DG, Schultz AB, Ashton-Miller JA, Alexander NB (1999). Age and gender differences in single-step recovery from a forward fall. J Gerontol 54(1): M44-M50. 